\title{
Sensitivity of troponins for diagnosis and risk prediction
}

\section{C6 \\ These findings enable much faster triage of patients}

Circulating levels of troponins can be indicative of myocardial injury, and the assays used to detect troponins are becoming ever-more sensitive. In three articles published in JAMA Cardiology, investigators report on the use of high-sensitivity assays for cardiac troponin I (hs-cTnI) to diagnose myocardial infarction (MI), and high-sensitivity assays for cardiac troponin $\mathrm{T}$ (hs-cTnT) to predict risk of coronary heart disease, heart failure, and death.

In a pooled analysis of five prospective, observational cohort studies, Edward Carlton et al. assessed 3,155 patients (56.1\% male, mean age 57.4 years) presenting with suspected acute coronary syndrome. Patients had a nonischaemic electrocardiogram determined, and hs-cTnI measured at presentation. "The specific aim of this investigation," says Carlton, "was to evaluate the diagnostic accuracy of a single 'one and done' high-sensitivity troponin testing strategy utilizing low cut-off values." The primary outcome (fatal or nonfatal acute MI occurring within 30 days of presentation) occurred in $9.2 \%$ of individuals. The lower limit of detection of hs-cTnI ( $1.2 \mathrm{ng} / \mathrm{l})$ had a sensitivity of $99.0 \%$ and a negative predictive value of $99.5 \%$ for acute MI. According to Carlton, "approximately $20 \%$ of patients may be suitable for discharge

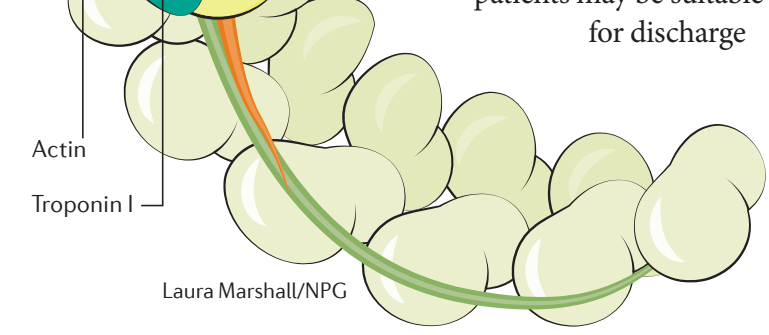

after a single blood test with a high diagnostic accuracy". As might be expected, higher cut-off thresholds reduced the sensitivity of the test and "may not have the diagnostic accuracy for clinical implementation".

Serial troponin measurements are recommended in ESC guidelines for diagnosis of MI at admission and after 3 h. Dirk Westermann et al. in the Biomarkers in Acute Cardiac Care study aimed "to challenge this approach using a more sensitive troponin assay with measurements at admission and after $1 \mathrm{~h}$ only". The investigators included 1,040 patients (64.7\% male, median age 65 years) presenting with acute chest pain. Application of a low hs-cTnI cut-off value $(6 \mathrm{ng} / \mathrm{l})$ after $1 \mathrm{~h}$ had a negative predictive value of $99.8 \%$, and a positive predictive value of $82.8 \%$ for non-ST-segment elevation MI type 1. The results were similar to those with a $3 \mathrm{~h}$ approach. Application of the $6 \mathrm{ng} / \mathrm{l}$ threshold resulted in lower mortality (1.0\%) during follow-up (median 313 days) compared with the routinely used $99^{\text {th }}$ percentile $(3.7 \%$; $P=0.001)$. "Acute MI can be ruled out in approximately $40 \%$ of all patients, while the diagnosis of acute MI can be made in $10 \%$," says Westermann. "These findings enable much faster triage of patients. We can now on the one hand rule out acute MI faster and discharge patients for further outpatient care; on the other hand, we can give the final diagnosis of acute MI much earlier, which gives us the option for faster revascularization in patients needing invasive care."

Troponin levels can also be used to predict risk of cardiovascular events in apparently healthy individuals. Almost all previous studies have evaluated the predictive value of a single measurement of troponin levels, whereas John W. McEvoy et al. investigated "whether repeat testing added further prognostic information". Measurements of hs-cTnT were taken 6 years apart from 8,838 participants in the Atherosclerosis Risk in Communities Study. Over 21 years of follow-up, incident detectable hs-cTnT levels (baseline $<5 \mathrm{ng} / \mathrm{l}$; follow-up $\geq 5 \mathrm{ng} / \mathrm{l}$ ) were independently associated with subsequent coronary heart disease (HR 1.4), heart failure (HR 2.0), and death (HR 1.5), relative to a hs-cTnT level $<5 \mathrm{ng} / \mathrm{l}$ at both visits. Individuals with the highest hs-cTnT levels at follow-up ( $\geq 14 \mathrm{ng} / \mathrm{l})$ had the greatest risks of cardiovascular disease, with hazard ratios of 8-12. "Our data suggest that two measurements are better than one and provide additional prognostic information," concludes McEvoy. Importantly, the investigators need to determine whether "the information gleaned from repeat troponin testing (perhaps in conjunction with B-type natriuretic peptide) can be used to effect clinical change in the preventive care of these patients and thereby reduce the burden of future disease".

Gregory B. Lim

ORIGINAL ARTICLES Carlton, E. et al. Evaluation of high-sensitivity cardiac troponin I levels in patients with suspected acute coronary syndrome. JAMA Cardiol. http://dx.doi.org/10.1001/ jamacardio.2016.1309 (2016) | Neumann, J. E. et al. Diagnosis of myocardial infarction using a high-sensitivity troponin I 1-hour algorithm. JAMA Cardiol. http://dx.doi.org/10.1001/ jamacardio.2016.0695 (2016) | McEvoy, J. W. et al. Six-year change in high-sensitivity cardiac troponin $T$ and risk of subsequent coronary heart disease, heart failure, and death. JAMA Cardiol. http://dx.doi.org/10.1001/jamacardio.2016.0765 (2016) 